\title{
MHD induced Fast-Ion Losses on ASDEX Upgrade
}

M. García-Muñoz ${ }^{1}$, H.-U. Fahrbach ${ }^{1}$, S.D. Pinches ${ }^{2}$, V. Bobkov ${ }^{1}$, M. Brüdgam ${ }^{1}$, M. Gobbin ${ }^{3,4}$, S. Günter ${ }^{1}$, V. Igochine ${ }^{1}$, Ph. Lauber ${ }^{1}$, M. J. Mantsinen ${ }^{1,5}$, M. Maraschek ${ }^{1}$, L. Marrelli ${ }^{3}$, P. Martin ${ }^{3,4}$, P. Piovesan ${ }^{3,4}$, E. Poli ${ }^{1}$, K. Sassenberg ${ }^{1,6}$, G. Tardini ${ }^{1}$, H. Zohm ${ }^{1}$ and the ASDEX Upgrade Team

${ }^{1}$ Max-Planck-Institut für Plasmaphysik, EURATOM Association, Garching, GERMANY

${ }^{2}$ Euratom/UKAEA Fusion Association, Culham Science Centre, Abingdon, OX14 3DB, UK

${ }^{3}$ Consorzio RFX, Associazione EURATOM-ENEA per la fusione, Padova, Italy

${ }^{4}$ Physics Department, University of Padova, Italy

${ }^{5}$ Helsinki Univ. of Technology, Association Euratom-Tekes, P.O.Box 4100, FIN-02015 HUT, Finland

${ }^{6}$ University College Cork, Association EURATOM-DCU, Cork, Ireland

e-mail: Manuel.Garcia-Munoz@ipp.mpg.de

Pacs: 52.25.Xz, 52.35.Bj, 52.55.Fa, 52.55.Pi, 52.55.Tn, 52.70.-m

\begin{abstract}
A detailed knowledge of the interplay between MHD instabilities and energetic particles has been gained from direct measurements of fast-ion losses (FIL). Time-resolved energy and pitch angle measurements of FIL caused by Neoclassical Tearing Modes (NTMs) and Toroidicity-induced Alfven Eigenmodes (TAEs) have been obtained using a scintillator based FIL-detector. The study of FIL due to TAEs has revealed the existence of a new core localized MHD fluctuation, the Sierpes mode. The Sierpes mode is a non-pure Alfvenic fluctuation which appears in the acoustic branch, dominating the transport of fast-ions in ICRF heated discharges. The internal structure of both, TAEs and Sierpes mode has been reconstructed by means of highly-resolved multichord soft X-ray measurements. A spatial overlapping of their eigenfunctions leads to a FIL coupling, showing the strong influence that a core-localised fast-ion driven MHD instability may have on the fast-ion transport. We have identified the FIL mechanisms due to NTMs as well as due to TAEs. Drift islands formed by fast-ions in particle phase space are responsible for the loss of NBI fast-ions due to NTMs. In ICRF heated plasmas, a resonance condition fulfilled by the characteristic trapped fast-ion orbit frequencies lead to a phase-matching between fast-ion orbit and NTM or TAE magnetic fluctuation. The banana tips of a resonant trapped fast-ion bounce radially due to an $E \times B$-drift in the TAE-case. The NTM radial bounce of the fast-ion banana tips is caused by the radial component of the perturbed magnetic field lines.
\end{abstract}

\section{Introduction}

The interplay between MHD instabilities and energetic particles is of special importance in burning plasmas with a large population of $\alpha$-particles like in ITER [1]. MHD instabilities can be driven by fast-ions but they also can lead to an enhancement of the fast-ions radial transport. In ITER, an $\alpha$-particle loss below 5\% of their nominal power is required to avoid grave consequences [2]. A detailed knowledge of the underlying physics can be gained from direct measurements of MHD induced fast-ion losses (FIL). ASDEX Upgrade (AUG) is well equipped for such studies, due to its powerful and flexible heating system (up to $20 \mathrm{MW} \mathrm{NBI}$ at $60 / 93 \mathrm{keV}$; up to 6MW ICRH and up to $2 \mathrm{MW}$ ECRH) and its well developed diagnostics. An array of magnetic pick-up coils (with $1 \mathrm{MHz}$ bandwidth) together with a set of SXR cameras (with $500 \mathrm{kHz}$ bandwidth) allow the study of the MHD fluctuation radial structure and helicity ( $\mathrm{n}$ and $\mathrm{m}$ mode numbers). A recently installed scintillator based FIL-detector (FILD) enables the identification of the MHD instability responsible for the FIL. The FILD design [3] follows the concept of the scintillator based $\alpha$-particle detector used for the first time in TFTR [4] and more recently in other fusion devices like CHS [5], W7-AS [6], LHD [7], JET [8], NSTX [9] 


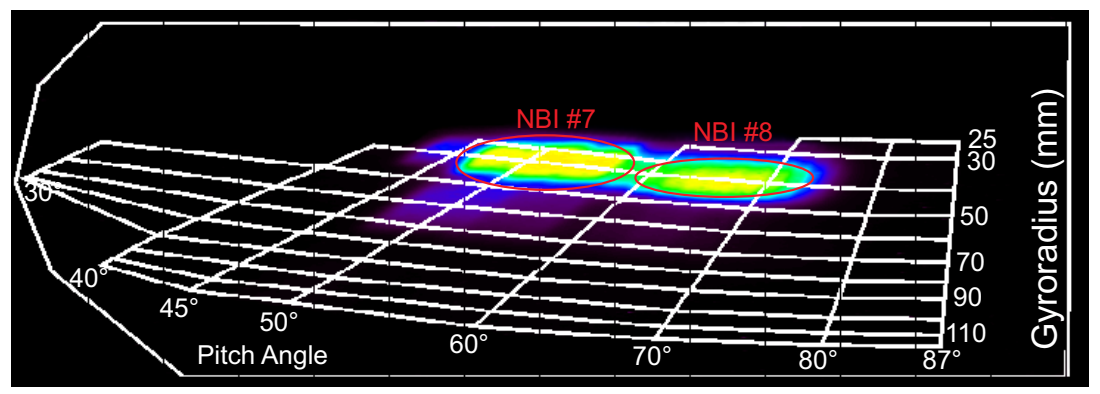

FIG. 1: AUG discharge \#21168: CCD frame showing fast-ion prompt losses of the NBI sources $\# 7$ and \#8 at $t=1.47 \mathrm{sec}$ in the FILD phase-space.

and TJ-II [10]. FILD acts as a magnetic spectrometer, dispersing fast-ions onto a scintillator, with their strike point depending on the gyroradius (energy) and pitch angle (angle between ion velocity and magnetic field line, $\Lambda=\operatorname{arcos}\left(v_{\|} / v\right)$ ). The active part of FILD, and main difference with respect to previous FILD designs, consists of a novel fast-response scintillator material with a decay time of $\approx 500 \mathrm{~ns}$ and a high quantum efficiency which allows identifying the MHD fluctuations responsible for the FIL through Fourier analysis (spectrograms). The detector head (collimator and scintillator plate) can be inserted via a movable manipulator up to a few mm behind the limiter, slightly above the mid plane.

In AUG, time-resolved energy and pitch angle measurements of FIL correlated in frequency and phase with NTMs [3, 11, 12] and fast-ion driven MHD instabilities [13] have been obtained using FILD. The study of FIL due to TAEs has revealed the existence of a new core localized MHD fluctuation, the Sierpes mode. The Sierpes mode is a non-pure Alfvenic instability which appears in the acoustic branch, dominating the transport of fast-ions in ICRF heated discharges. The internal structure of both, TAEs and Sierpes mode has been reconstructed by means of highly-resolved multichord soft X-ray measurements. A spatial overlapping of their eigenfunctions leads to a FIL coupling, showing the strong influence that a core-localised fast-ion driven MHD instability may have on the fast-ion transport. We have identified the FIL mechanisms due to NTMs as well as due to TAEs. Drift islands formed by fast-ions in particle phase space are responsible for the loss of NBI fast-ions due to NTMs. In ICRF heated plasmas, a resonance condition fulfilled by the characteristic trapped fast-ion orbit frequencies lead to a phase-matching between fast-ion orbit and NTM or TAE magnetic fluctuation.

The theoretical investigation of the mechanisms leading to the expulsion of fast-ions (e.g. $\alpha$ particles) is nowadays becoming more relevant as we need to validate the numerical tools for ITER predictions. Further efforts to simulate the loss of fast-ions due to MHD instabilities are needed, since the calculated MHD induced fast-ion transport, so far, is up to one order of magnitude smaller than the measured losses [14]. FILD, the improved set of fluctuation diagnostics like magnetic pick-up coils, SXR-cameras and Doppler reflectometry, and the, for this purpose developed numerical tools should clarify such discrepancies in the FIL levels. In this paper, we present the observation and preliminary modeling of FIL due to NTMs and fast-ion driven MHD instabilities in AUG. The end of each section is devoted to discuss the implications of these results for ITER.

\section{Fast-ion losses in MHD-quiescent plasmas}

External heating systems may cause the prompt loss of fast-ions directly through their heating mechanisms. They can inject fast-neutrals (NBI systems) which get ionized on trajectories within the loss cone but they also can actively enhance the loss of fast-ions by accelerating 
a)

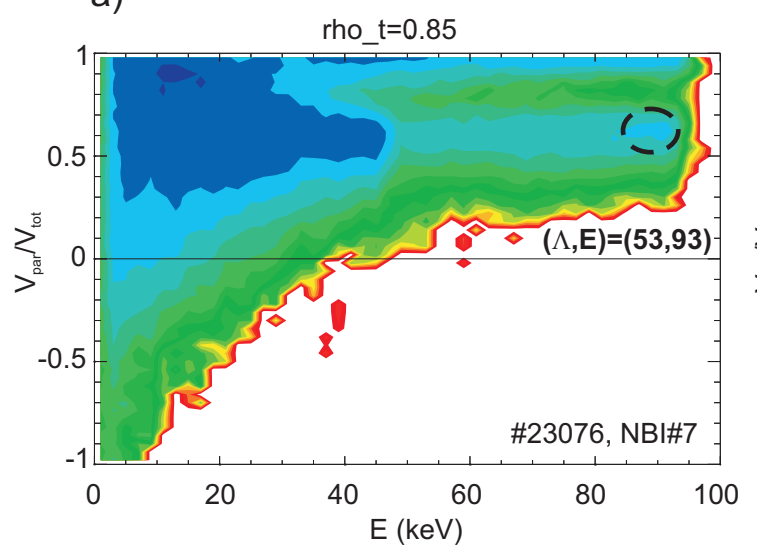

b)

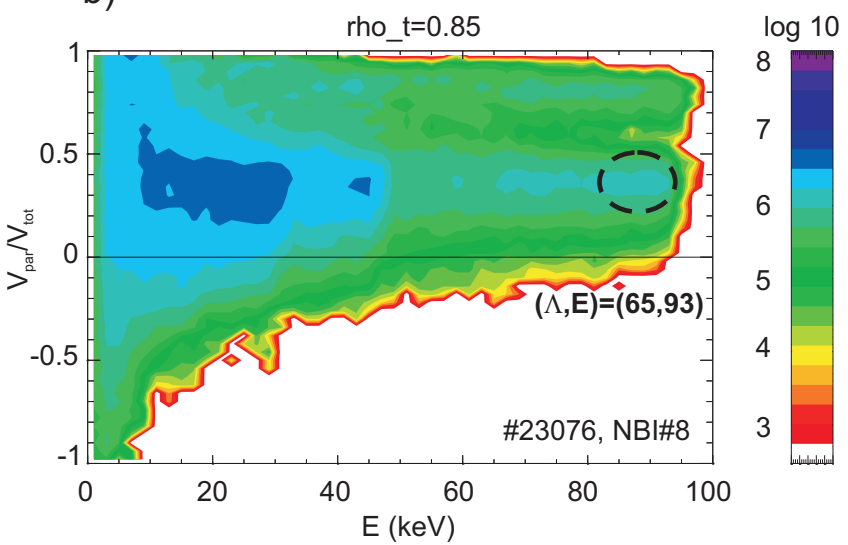

FIG. 2: AUG discharge \#23076: Contour plots of TRANSP simulation of NBI fast-ion distribution in $(\Lambda, E)$ space generated by NBI sources \#7 (a) and \#8 (b) at $\rho_{t}=0.85$.

preferential velocity components (ICRF systems). In fact, fast waves push fast-ions across the loss boundary into the loss cone by accelerating the perpendicular velocity component. The results of NBI and ICRH prompt loss studies carried out with the FILD system are presented briefly in this section.

\subsection{Neutral Beam Injection}

Beams of fast neutrals are commonly used in magnetic fusion devices to heat (or diagnose) the plasma, to drive current and to study MHD instabilities. Depending on the final goal of the beam, different injection geometries and energies are applied. Intrinsic to the NBI operation are the fast-ion prompt losses, open trajectories (within the loss cone) originated near to or in the Scrape Of Layer (SOL) which intersect in-vessel components. The energy, pitch angle and total amount of prompt losses depend on the main plasma parameters and the NB-injection and $3 \mathrm{D}$ vacuum vessel geometry. The AUG NBI system has $8 \mathrm{NBI}$ sources with different injection angles, each one capable of supplying 2.5 MW, producing, therefore, diverse ionization profiles of fast-neutrals. This rich and reliable pattern of NBI prompt losses has been used to validate the FILD energy-pitch angle grid. Fig. 1 shows the prompt loss pattern produced by two NBI sources on the scintillator plate. Both sources inject neutral deuterons with a maximal energy of $93 \mathrm{keV}$ i.e. a gyroradius of $\approx 40 \mathrm{~mm}$ for a magnetic field of $B_{t}=1.6 T$ at the probe. While source \#7 injects neutrals rather tangential in plasma, source \#8 does it more radially. Accordingly, source \#7 produces ion prompt losses with lower pitch angles, $\Lambda \approx 50^{\circ}$ than source $\# 8, \Lambda \approx 65^{\circ}$. TRANSP [15] simulations have been carried out to investigate the fast-ion distribution function generated by each NBI source. Fig. 2 shows the fast-ion distribution functions generated by the most intense NBI sources in FILD, \#7 and \#8, at $\rho_{\text {tor }}=0.85$. TRANSP predicts, for the main injection energy, a pitch angle maximum in the fast-ion distribution function at $\Lambda \approx 53^{\circ}$ for NBI source \#7 and at $\Lambda \approx 65^{\circ}$ for NBI source \#8 which is in excellent agreement with FILD measurements, see Fig. 1. The NBI prompt loss pattern on the first wall of the vacuum vessel is a fully $3 \mathrm{D}$ issue. In-vessel protruding components, NBI injection geometry and energy, 3D magnetic equilibrium and plasma parameters, especially $n_{e}$ and $T_{e}$ in the SOL, determine the poloidal and toroidal distribution of NBI prompt losses on the wall. Therefore, not all NBI sources in AUG are detectable by FILD, in fact, only those injecting ions on trajectories connected with FILD can be detected. 


\subsection{Ion Cyclotron Resonance Heating}

In present fusion plasma devices, fast-ions generated by ICRH are used to heat the plasma, to drive current, to study MHD instabilities and to some extent to simulate and investigate the physics of fusion-born $3.5 \mathrm{MeV} \alpha$-particles in a fusion reactor. Fast waves launched into the plasma at the ion gyrofrequency or at its harmonics accelerate the ion gyromotion as the ions pass through the rather vertical resonance layer. This heating mechanism tends to produce energetic ions on trapped orbits with their banana tips within the resonance layer. A highly anisotropic distribution function is formed with up to $90 \%$ of the total ion energy in the perpendicular component. The losses of fast-ions from the ICRH distribution function tail in MHD-quiescent plasmas appear when the ion orbit width is comparable to the plasma minor radius [16]. The ICRH induced FIL are toroidally symmetric and poloidally localized above or below the mid-plane depending on the ion grad-B drift.

The AUG ICRF system is capable of launching fast waves into the plasma with a heating power of up to $6 \mathrm{MW}$ in a multifrequency fashion (30-62 MHz) allowing a rich variety of heating schemes. In the most typical AUG magnetic configuration, with downwards ion grad-B drift, no losses of ICRH fast-ions have been observed so far with the FILD system in MHD quiescent plasmas. Although the ICRH mechanism is supposed to enhance the radial transport of fastions, their losses might be located below the mid-plane, while FILD is at $\approx 33 \mathrm{~cm}$ above the mid-plane. However, the fast-ion transport due to the ICRF heating itself must be taken into account to understand the fast-ion confinement in ICRF heated plasmas with MHD activity.

Fig.3 illustrates the ICRF heating mechanism and induced transport. Starting with thermal deuterium ions, e.g. a passing ion with $(\Lambda, \mathrm{E})=\left(10^{\circ}, 5 \mathrm{keV}\right)$, fast waves accelerate the perpendicular component of the ion velocity as they pass through the resonance layer, trapping the ions in a banana orbit. The second trajectory plotted in Fig. 3 shows the next stage of the ICRF heating mechanism, a barely trapped deuterium ion with $(\Lambda, E)=\left(63^{\circ}, 30 \mathrm{keV}\right)$. As the trapped ions keep on passing through the ICRH resonance layer, the heating mechanism itself pushes the banana tips outwards, in Fig. 3 right- and upwards. This is illustrated in the following two ion trajectories; trapped ions with $(\Lambda, E)=\left(75^{\circ}, 200 \mathrm{keV}\right)$ and $(\Lambda, E)=\left(79^{\circ}, 300 \mathrm{keV}\right)$. As the ions gain energy, the banana orbit width also gets broader and simultaneously their banana tips explore the plasma minor radius outwards and so all MHD-instabilities along the q-profile.

\section{Fast-Ion Losses due to NTMs}

It is well known that a significant fraction of plasma pressure in a magnetically confined fusion plasma is carried by fast-ions. While the NTM impact on the global confinement is rather well established, less is known on how they influence energetic particles, like for example ICRF heated ions, or ions of NBI origin. Experiments on this subject have been performed in TFTR [17] and DIII-D [18] using $\alpha$-particles and NBI fast-ions respectively. A good understanding of the magnetic island effects on the fast-ion confinement is important, for example, to fully assess the efficiency of external heating systems and NBI current drive [19]. In next generation devices, like ITER, this information is important also to predict the confinement of $\alpha$-particles and the impact of their losses on plasma facing components. The AUG heating system allows a rich variety of scenarios, where the behaviour of fast-ions can be finely tuned and decoupled from the bulk plasma environment. Dedicated studies to investigate the effect of NTMs on the fast-ions confinement of NBI and ICRF origin have been carried out separately. 


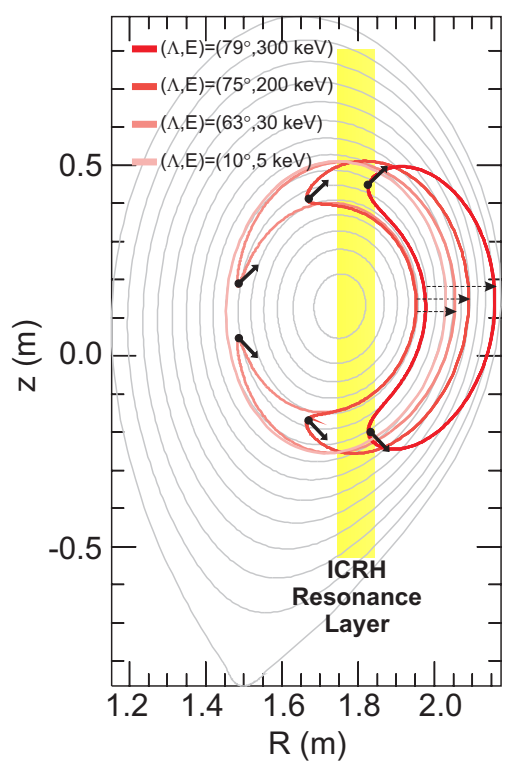

FIG. 3: Schematic of the ICRF heating and loss mechanism.

\subsection{NTM Induced Fast-Ion Losses of NBI Origin}

The loss of NBI fast-ions correlated in frequency and phase with a $(2,1)$ magnetic island fluctuation has been measured with the FIL-detector and interpreted by means of a numerical model $[3,11]$. The $(2,1)$ NTM contribution to the measured FIL pattern is twofold; an enhancement of the NBI prompt loss flux and a loss of passing fast-ions which are thought to be well confined in the absence of magnetic fluctuation.

The experiments discussed in this section have been mainly performed in plasmas with toroidal current $I_{p}=0.8 \mathrm{MA}$, toroidal field $B_{t}=2 \mathrm{~T}$, safety factor at the edge $q_{95}=4.5$ and NBI as main heating and fast-ion source. The intermittent operation of an NBI source (2.5 MW), with a switch-off time shorter than $50 \mu s$, has been used to provide a periodically changing source of fast-ions which helps studying the loss time scales. The plasma density is kept at a relatively low value of $4.5 \times 10^{19} \mathrm{~m}^{-3}$ during the NBI modulation phase. An NBI heating ramp-up (up to $15 \mathrm{MW}$ ) was performed to trigger a $(2,1)$ magnetic island. During the modulation of the NBI source a constant heating power of $5 \mathrm{MW}$ was kept. Fig.4(a) shows the magnetic activity due to the $(2,1)$ magnetic island. A Fourier analysis of the FIL-flux with pitch angles, $\Lambda=(35,45)^{\circ}$, corresponding to passing orbits is shown in Fig.4(b). Besides the slow trend following the frequency evolution of the mode and of its harmonics, we note discrete spots corresponding to the NBI modulation. As shown in Fig.4(c), the amplitude of the losses at the dominant mode frequency (black curve) is modulated according to the NBI evolution (red curve) and its envelope follows the amplitude of the magnetic fluctuation (blue curve). Two well distinguished FIL time scales, depending on the pitch angle of the lost ions, have been observed experimentally by modulation of the NBI source. Losses of fast-ions with rather parallel velocities, $\Lambda=(35,45)^{\circ}$, decay promptly as soon as the modulated NBI source is switched off, thus indicating a time scale for these losses not longer than a few tens of $\mu s$. In contrast, the loss of fast-ions with rather perpendicular velocity, $\Lambda=(70,75)^{\circ}$, needs $\approx 5 \mathrm{~ms}$ to vanish after the NBI switching off. The typical lost ion flux varies from $4 \cdot 10^{13} \frac{\mathrm{Ions}}{\mathrm{s} \cdot \mathrm{cm}^{2}}$ for the NTM prompt losses up to $10^{14} \frac{\mathrm{Ions}}{\mathrm{s} \cdot \mathrm{cm}^{2}}$ for the lost ions with higher pitch angle. Both signals are comparable to the maximal NBI prompt loss signal, $4 \cdot 10^{14} \frac{\mathrm{Ions}}{\mathrm{s} \cdot \mathrm{cm}^{2}}$. The observed strong increase of FIL caused by magnetic islands is consistent with the heat load to the limiters measured in plasmas as soon 


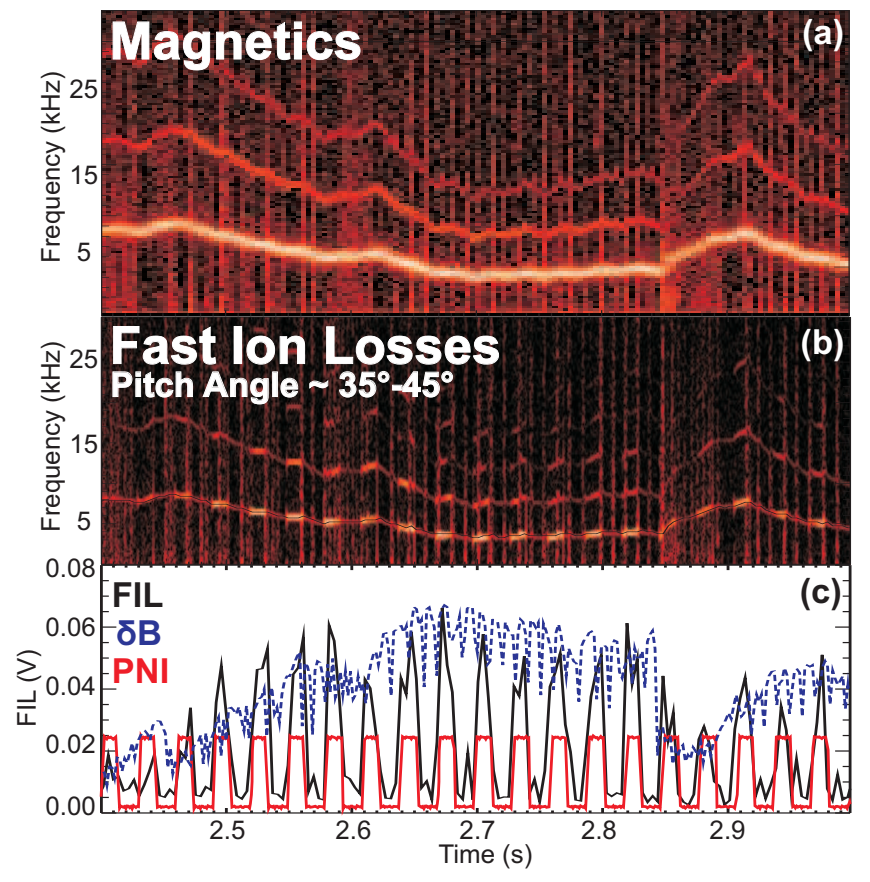

FIG. 4: AUG discharge \#21168: (a) and (b): spectrograms of the magnetic island fluctuation and FILD signal corresponding to losses with pitch angle $\Lambda=(35,45)^{\circ}$ respectively. $(c)$ : waveforms of the NBI modulated power (red curve; modulation amplitude of $2.5 \mathrm{MW}$ ), of the amplitude of the $(2,1)$ mode (blue doted curve) and of the amplitude of the FILD signal at the frequency of the $(2,1)$ magnetic fluctuation (black curve). Based on Ref.[3].

as large magnetic islands appear.

Drift orbit calculations using the ORBIT [20] and the GOURDON [21] codes have allowed the identification of the loss mechanisms. The intersection of the drift islands formed by fastions in particle phase-space with plasma-facing components is responsible for the prompt NTM induced FIL (loss time of $\approx 10 \mu s$ ) [22]. An overlap of these drift islands leads to an orbit stochastization and consequently to a loss of fast-ions on a diffusive time-scale (loss time $\approx$ few ms) $[22,23]$. This overlap depends on the shape of the q-profile, on the location of the $q=2$ resonance surface, the amplitude of the original $(2,1)$ mode and the fast-ion orbital drift characteristics.

Dedicated experiments are foreseen in AUG to estimate the effect of magnetic islands with $(2,1)$ helicity on $\alpha$-particle-like fast-ions in ITER-like scenarios with a $q=2$ surface rather close to the separatrix which could lead to a strong increase of the $3.5 \mathrm{MeV} \alpha$-particle loss due to orbit stochasticity.

\subsection{NTM Induced Fast-Ion Losses of ICRF Origin}

Losses of ICRF generated trapped fast-ions caused by the presence of low-frequency $(3,2)$ NTMs have been observed and investigated numerically [12] with the HAGIS code [24], v8.09. The experiments discussed here were performed in plasmas with toroidal plasma current $I_{p}=$ 1.2 MA, toroidal magnetic field ramp of $B_{t}=1.95-2.15 \mathrm{~T}$, and safety factor at the edge $q_{95}=3.2$. Auxiliary plasma heating was provided by means of two NBI sources delivering a total power of $5 \mathrm{MW}, 1 \mathrm{MW}$ of ECRH and $3 \mathrm{MW}$ of on-axis ICRF hydrogen minority heating in a deuterium plasma with a hydrogen to deuterium ratio of $n_{H} / n_{D} \approx 6 \%$. Fig.5(a) shows the relevant plasma parameters for the reference discharge, \#21081. The MHD activity during the discharge is shown in Fig.5(b) through a spectrogram of a magnetic fluctuation pick-up 

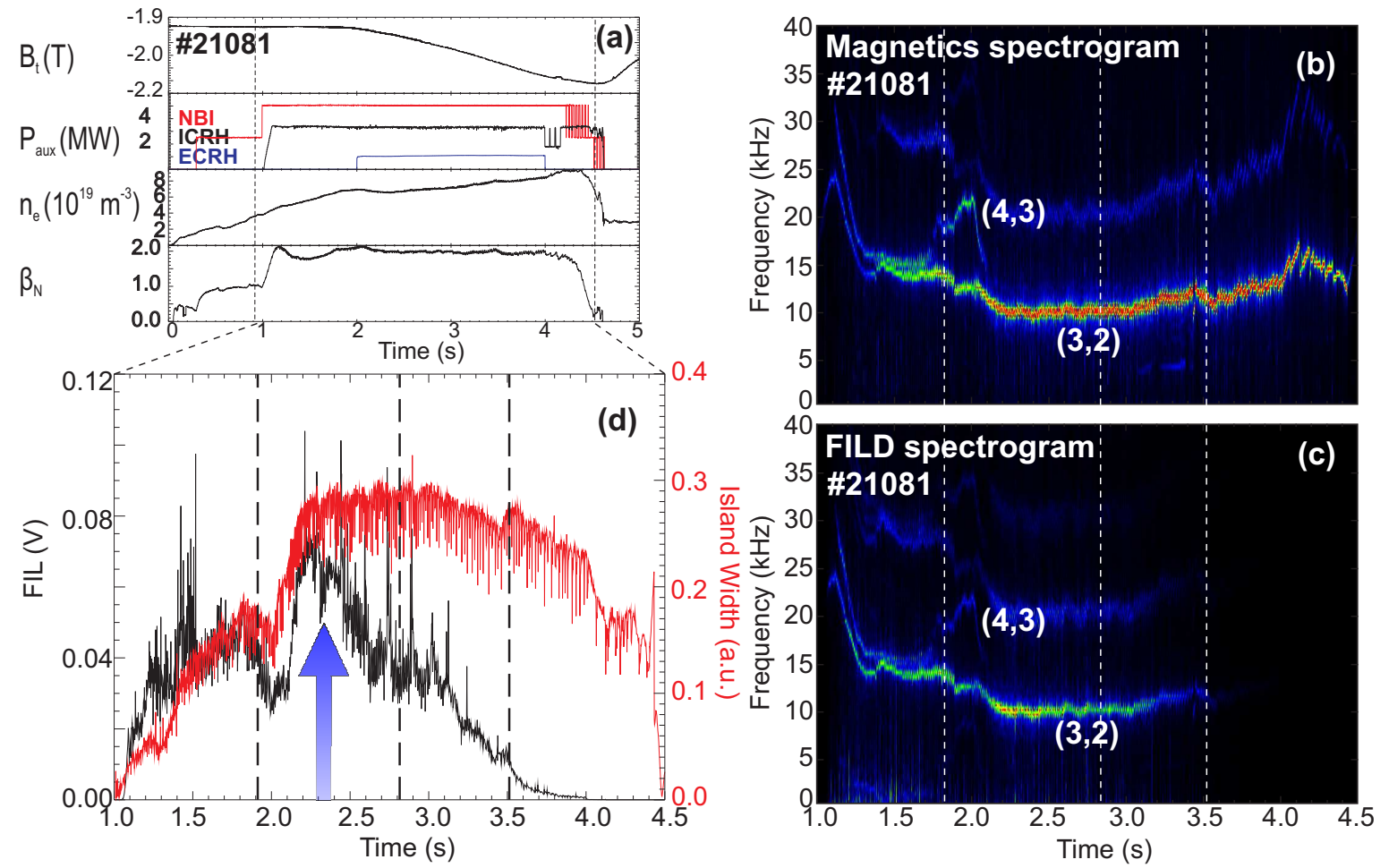

FIG. 5: AUG discharge \#21081: (a) Overview of the discharge; from top to bottom, the evolution of the toroidal magnetic field, heating power, core electron density, and $\beta_{N}$ are presented. (b) magnetic spectrogram showing the MHD activity. (c) FILD spectrogram of lost ions with gyroradius $\rho \approx 50 \mathrm{~mm}$. (d) Amplitude analysis of $(3,2)$ magnetic island and FILs at the fluctuation frequency. The $B_{t}$ ramp onset is highlighted with a vertical blue arrow. Adapted from Ref.[12].

coil signal. The presence of a $(3,2)$ NTM is clearly visible during the whole time window while the secondary magnetic island with $(4,3)$ helicity disappears at $t=2.2 \mathrm{~s}$. EC waves were launched with the goal of stabilizing the NTM (in this shot, however, the correct matching of the EC resonance with the island position through the toroidal magnetic field $\left(B_{t}\right) \mathrm{ramp}$ did not succeed). In order to identify the lost particles in phase-space we analyze the loss pattern recorded by the CCD camera of the FIL-detector during the MHD activity.

Fig.6 shows the evolution of FIL in phase-space for the AUG discharge \#21081 during the magnetic islands activity and $B_{t}$ ramp. The losses of fast-ions induced by the $(4,3)$ and $(3,2)$ magnetic islands together with the prompt losses generated by both NBI sources, \#3 and \#7, at $t=1.83 \mathrm{~s}$ are shown in Fig.6(a). The prompt losses from the more radially injected ions (NBI source \#3) are barely visible in the region of higher pitch angle, $\Lambda \in(70,75)^{\circ}$, while a tangential NBI source (\#7) produces prompt losses in a lower pitch angle region, $\Lambda \in(45,55)^{\circ}$. When both magnetic islands are present, two different contributions to the FIL pattern are simultaneously visible at different gyroradii and almost the same pitch angle. For the magnetic field of $1.6 T$ at the probe, the losses peak at a gyroradius of $50-55 \mathrm{~mm}$, correspond to hydrogen ions with energies $E_{H}$ between 250 and $600 \mathrm{keV}$, and pitch angles $\approx 64^{\circ}$. The losses at larger gyroradii, between 60 and $110 \mathrm{~mm}$, correspond to hydrogen ions with energies, $E_{H}$, between 1.0 and $1.5 \mathrm{MeV}$ and pitch angles between $62^{\circ}$ and $65^{\circ}$. The next frame, Fig.6(b), shows the losses of fast-ions measured by FILD when only the $(3,2)$ NTM is present. The contribution of the $(3,2)$ magnetic island to the FIL pattern appears at gyroradii of 50-55 $\mathrm{mm}$ while the losses at larger gyroradii disappear in the absence of the $(4,3)$ magnetic island. The FIL due to the $(3,2)$ 
a)

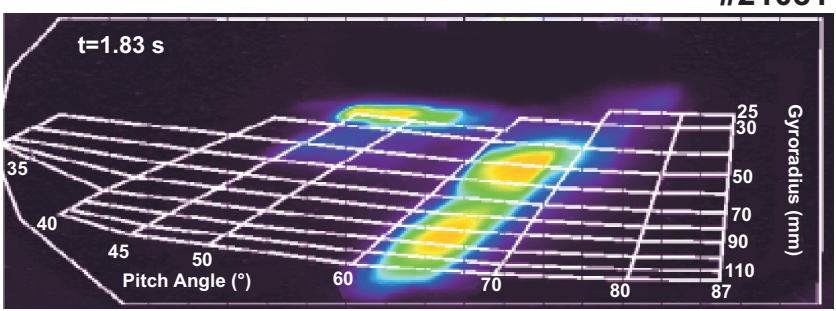

b)

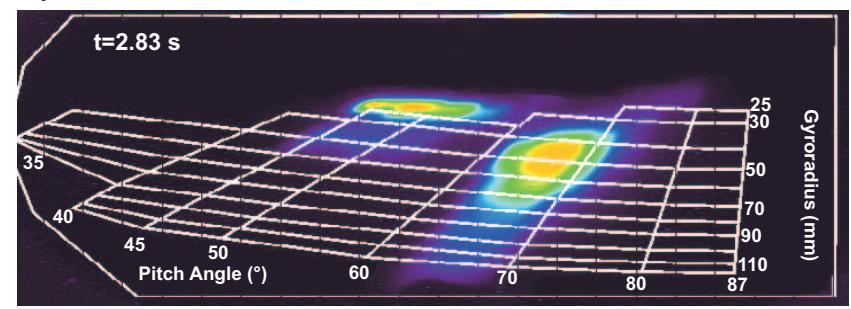

c)

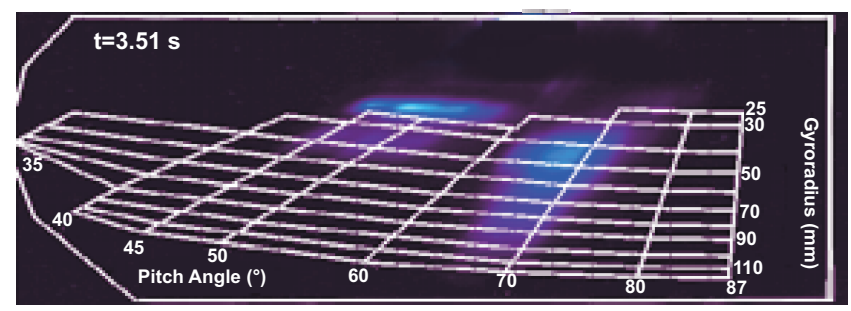

FIG. 6: AUG discharge \#21081: CCD view of the light pattern produced by the incident ions ejected from the plasma due to interactions with magnetic islands.

magnetic island disappear during the $B_{t}$ ramp at a constant magnetic island width, see Fig.6(c). The ratio of FIL versus magnetic island width has been investigated by means of a Fourier analysis. Fig.5(c) shows an FFT analysis of the FIL signal presented in Fig.6(a). The similarity in the magnetics and FILD spectrograms are striking. The FILD spectrogram is not only revealing the loss of fast-ions due to the $(3,2)$ magnetic island, but also due to $(4,3)$. The $(3,2)$ island width is shown in Fig.5(d) together with the amplitude of the FIL signal at the $(3,2)$ magnetic fluctuation frequency. The FIL-flux depends sensitively on the relative radial location of the fast-ion population with respect to the magnetic island radial position. A shift of the ICRH resonance layer by means of the $B_{t}$-ramp leads to a large decrease in the FIL-flux, as shown in Fig.5(d).

The fast-ion expulsion is explained in terms of the radial drift induced by the perturbed magnetic field, $\tilde{\mathbf{B}}$, when the resonance condition $\Omega_{n, p}=n \omega_{\phi}-p \omega_{\theta}-\omega \approx 0$ is fulfilled, where $n$ is the toroidal mode number, $p$ is the poloidal harmonic, $\omega_{\phi}$, the fast-ion precession frequency, $\omega_{\theta}$, the fast-ion poloidal frequency and $\omega$ the mode frequency [25]. At the high energies we are considering, $\omega_{\phi}$ and $\omega_{\theta}$ become comparable, and since they are much larger than $\omega$, the resonance condition can be satisfied if $n \omega_{\phi} \approx p \omega_{\theta}$. In general, the radial drift induced by a MHD mode is a combination of the $E \times B$-drift and the radial excursion following the magnetic field lines caused by the perturbed field, $\tilde{\mathbf{B}}$. In the presence of a quasistatic $\left(\omega \ll \omega_{\phi}, \omega_{\theta}\right)$ magneticfield fluctuation, the dynamics of the trapped particles is changed essentially in two ways: a radial component in the parallel velocity appears and the parallel gradient in the mirror force is modified. The role of the electric field associated to the mode, which scales proportionally to the mode frequency, $\omega$, is negligible. In the experiments described above, where $n=2$, the resonance condition $2 \omega_{\phi} \approx \omega_{\theta}$ is satisfied [12]. In other words, the particle has the same phase 


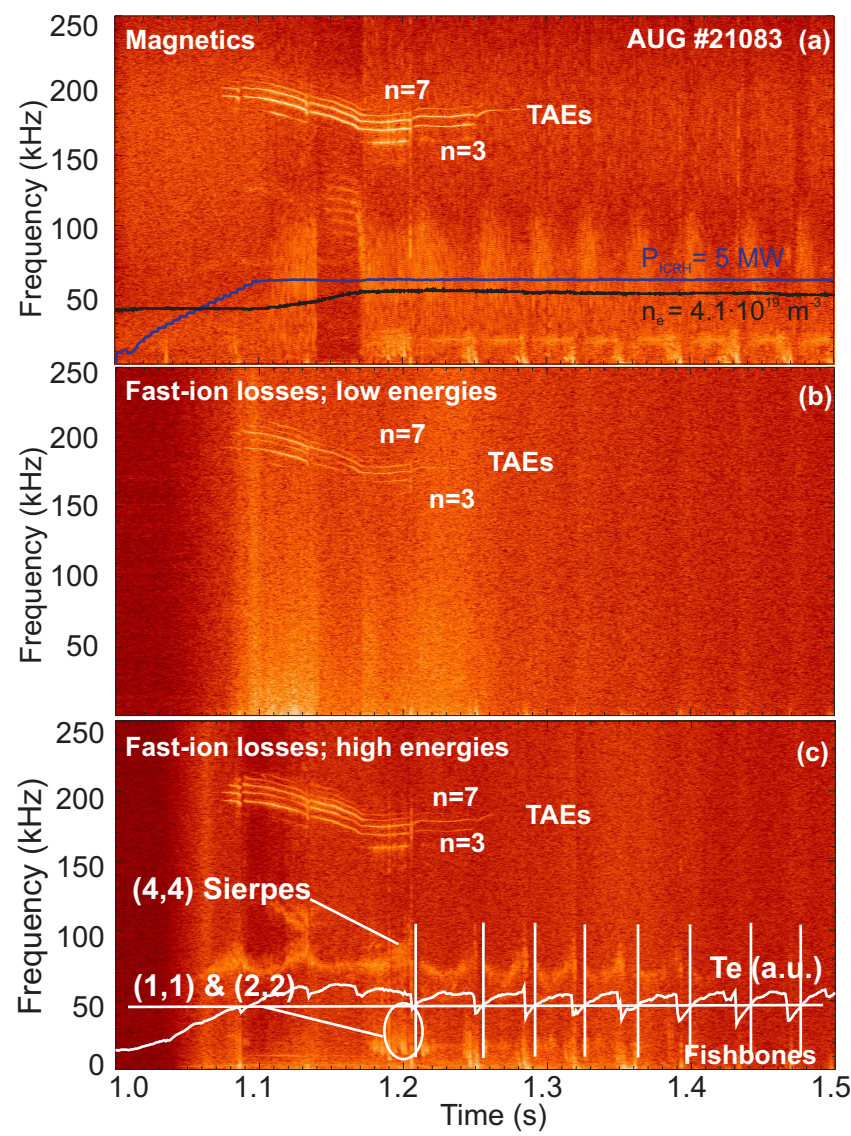

FIG. 7: AUG discharge \#21083: Spectrograms of magnetic fluctuation (a) and FIL of low (b) and high (c) energies. Adapted from Ref.[13]. 
with respect to the island after each bounce time. This leads to a secular radial drift motion, with its direction, inwards or outwards, being determined by the relative alignment of the parallel velocity and of the magnetic-field perturbation. This explains the phase locking observed in the measurements, where the FILs are modulated at the mode rotation frequency and occur during half of the rotation period.

In ITER, $3.5 \mathrm{MeV} \alpha$-particles will have a poloidal motion frequency at about midradius of $\omega_{\theta} / 2 \pi \approx 460 \mathrm{kHz}$ and a toroidal precession frequency $\omega_{\phi} / 2 \pi \approx 115 \mathrm{kHz}$, indicating a potential resonance with quasistatic $n=4$ magnetic islands. Furthermore, the impact of a magnetic island chain with different helicities must be asset.

\section{ICRH Fast-Ion Losses due to Fast-Ion Driven MHD Instabilities}

Energetic particles often drive MHD instabilities unstable in magnetically confined fusion plasmas. Two kinds of fast-ion driven MHD instabilities can be distinguished; Alfven Eigenmodes (AEs) which are part of the continuum spectrum and appear in toroidally confined plasmas because of the periodicity in the refraction index [26], e.g. Toroidal Alfven Eigenmodes (TAEs) [27-29], and energetic particle modes (EPMs) which appear usually at a characteristic frequency of the fast-ion orbits when the energetic particle pressure is comparable to the plasma pressure [30]. An energy exchange between particle and wave takes place if an amount of free energy is available through gradients in the fast-ion distribution function and a wave-particle resonance condition is fulfilled. The resonance condition between the wave phase velocity and the fast-ion orbital frequencies already has been introduced in the previous section to explain the expulsion of ICRH fast-ions due to NTMs. On one hand, particles may transfer energy to the waves overcoming the continuum and background plasma damping and driving them unstable. On the other hand waves may transfer energy to the particles leading to a radial drift and a possible loss. The fast-ion driven MHD stability, drive and damping rates [31, 32], and the subsequent fast-ion transport is an issue of great interest since the large fast-ion pressure in ITER is expected to drive MHD fluctuations unstable in all foreseen scenarios [33]. In particular, the loss of fast-ions due to TAEs [34-37] and EPMs [38, 39] has been extensively studied in most large fusion devices due to their ability to affect the fusion performance and cause severe damages to the plasma facing components [40]. However, most of these studies are either based on neutron drops correlated with bursting MHD instabilities or are lacking of the necessary timeresolved internal fluctuation structure or pitch angle and energy measurements of the lost ions. Recent breakthroughs in plasma diagnostic capabilities have allowed accurate measurements of the internal structure of AEs [41] as well as their induced fast-ion redistribution [14] and loss $[13,42]$.

In this section we present the observations and modeling of FIL due to fast-ion driven MHD instabilities in ICRF heated AUG plasmas. The nature of a recently discovered fast-ion driven MHD instability, the Sierpes mode [13], is briefly discussed here and will be the subject of a forthcoming detailed publication.

The experiments discussed here have been mainly performed in plasmas with toroidal plasma current $I_{p}=1.0-1.2 \mathrm{MA}$, toroidal field $B_{t}=2.0-2.2 \mathrm{~T}$, safety factor at the edge $q_{95}=$ $3.2-4.0$ and ICRH as main heating and fast particle source. $5 \mathrm{MW}$ of on-axis ICRH of hydrogen minority was applied in a deuterium plasma $\left(n_{H} / n_{D} \approx 6 \%\right)$. Fig.7(a) shows the typical core line averaged electron density, $\bar{n}_{e}$, together with ICRH power for a reference discharge, $\# 21083$, overploted on a Fourier spectrogram of a magnetic fluctuation signal. Several TAEs with frequencies between 150 and $225 \mathrm{kHz}[43,44]$ and toroidal mode numbers $n=3,4,5,6,7$ are clearly visible at $t \approx(1.0-1.3) \mathrm{s}$. At lower frequencies, up to $25 \mathrm{kHz}$, some bursting fishbone modes [45] appear. 


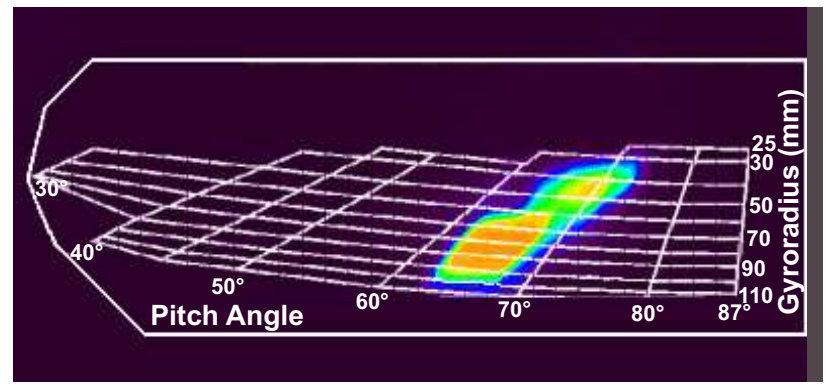

FIG. 8: AUG discharge \#21011: CCD view of the light pattern produced by the incident ions ejected from the plasma due to interactions with high frequency modes, Ref.[13].

The energy and pitch angle of the FIL due to these MHD instabilities are presented in Fig. 8, which shows a CCD frame for the discharge \#21011 at $t=1.43 \mathrm{~s}$. When the TAEs are present, two different contributions to the FIL pattern are simultaneously visible at different gyroradii and almost the same pitch angle. For the magnetic field of $1.6 T$ at the probe, the losses peak at a gyroradius of $45 \mathrm{~mm}$, correspond to hydrogen ions with $E_{H} \approx 250 \mathrm{keV}$, and pitch angles between $68^{\circ}-70^{\circ}$. The losses at higher energies appear with a much broader distribution in giroradii, between 60 and $110 \mathrm{~mm}$ which correspond to hydrogen ions with $E_{H} \approx 1 \mathrm{MeV}$ and pitch angles between $62^{\circ}$ and $68^{\circ}$.

In order to identify the MHD instabilities responsible for these losses, a Fast Fourier Transformation (FFT) was applied to the signal of the photomultipliers which observe the phase-space regions where losses are detected. Fig. 7(b) shows the spectrogram of a signal, which is measuring lost ions with a gyroradius $\approx 45 \mathrm{~mm}$ (upper spot in Fig. 8). We observe a correlation between the frequency and phase of the individual TAEs $(n=3,4,5,6,7)$, see Fig. 7(a)-(b), and those of the losses. The spectrogram in Fig. 7(c) refers to ion losses at larger gyroradii $(60-110 \mathrm{~mm})$, i.e. the lower spot in Fig. 8. A clear correlation between the TAE frequency pattern and the FIL frequencies is also observed. An interesting feature is present in the FILD spectrogram of high energies at frequencies within the Alfven-acoustic branch, $\approx 80 \mathrm{kHz}$, where a dominant pattern emerges. We call this new MHD instability the Sierpes mode because of its footprints in the FIL spectrogram, its deleterious effect on the fast-ion confinement and the fact that it is hardly visible for the Mirnov pickup coils, see Fig. 7(a). Tracking the frequencies corresponding to the individual TAEs, we observe stronger losses (up to a factor of three higher) due to TAEs if the Sierpes mode is also ejecting fast-ions. This can be observed by comparing the losses due to individual TAEs in both FILD channels.

\section{The Sierpes mode}

The stability of the Sierpes mode is more weakly dependent on background plasma parameters than the TAEs, remaining unstable usually for time periods much longer than the TAEs, eventually up to $1 \mathrm{sec}$, unaffected by changes in the $q$-profile. The Sierpes fluctuation always disappears at large sawtooth crashes and appears again within the next $\approx 10 \mathrm{~ms}$, when the $T_{e}$, i.e. the collisionality $\left(\mathrm{v}_{e}\right)$ has reached a certain threshold, $T_{e}=1.9 \mathrm{keV}$, and the fast-ion population has been rebuilt. The frequency of the Sierpes mode, $f_{\text {Sierpes }}$, does not change with the toroidal magnetic field $B_{t}$ or the core electron density $n_{e}$, see Fig. 7(c). A rapid change of the electron density, $n_{e}$, due to L-H mode confinement transition, $t \approx 1.15 \mathrm{sec}$ in Fig. 7 (c), is followed by a change in the $f_{T A E}$, as expected, but not by a change in $f_{\text {Sierpes. }}$. This suggests that the Sierpes mode is not a pure Alfvénic mode. Furthermore, the rapid frequency rise before the sawtooth 


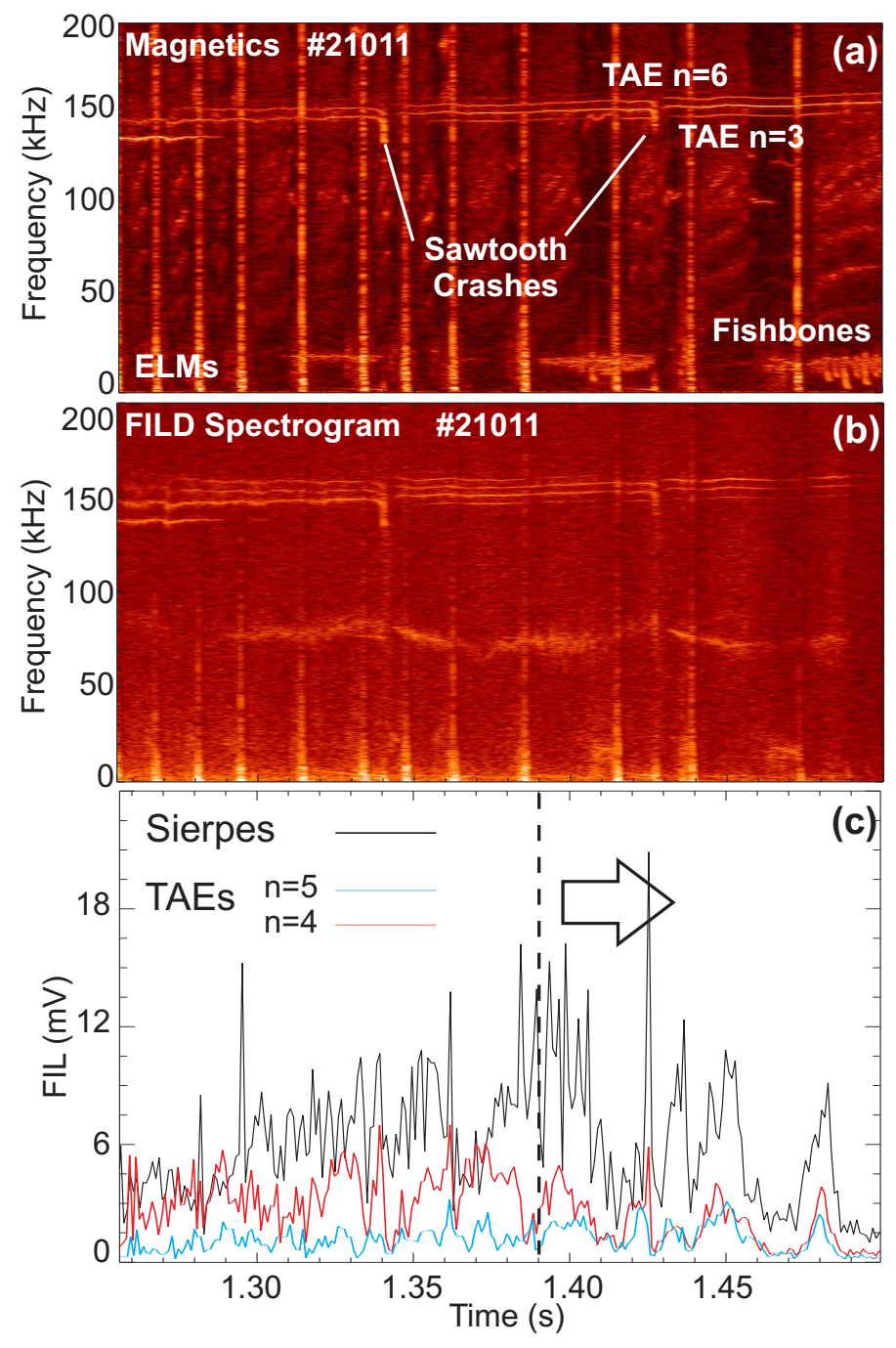

FIG. 9: AUG discharge \#21011. (a) spectrogram of an in-vessel magnetic pick-up coil. (b) spectrogram of the FILD FIPM12 channel showing fast ion losses correlated with various types of MHD activities i.e. TAEs, Sawtooth crashes, ELMs and the Sierpes mode. (c) frequency tracking of two individual TAEs, $n=4$ and $n=5$, and the Sierpes mode. Adapted from Ref.[13]. 


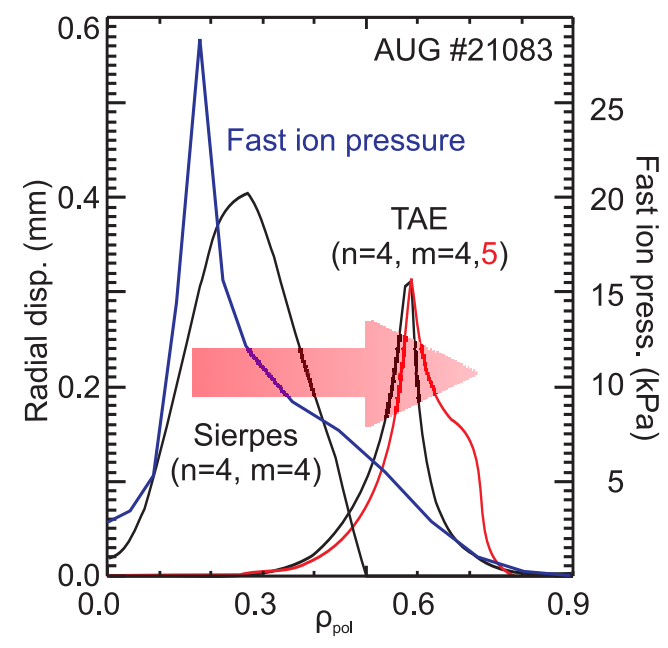

FIG. 10: Schematic of the fast-ion channeling due to a radial chain of multiple fast-ion driven MHD fluctuations.

crashes can not be explained by the classical Alfvénic physics since no relevant background plasma parameter (i.e. $n_{e}$ or $B_{t}$ ) change so fast. In fact, the behavior before the sawtooth crash suggests some kinetic effects of the energetic particles as in the EPM case. Including acoustic coupling on the shear Alfven spectrum allows the mixing of the acoustic and Alfven branches in the lower Alfven continuum. Modes within this branch like the Beta-induced Alfven Eigemodes (BAEs) [46, 47] or Beta-induced Alfven Acoustic Eigenmodes (BAAEs) [48] are neither pure Alfvenic nor pure acoustic with a dispersion relation proportional to the sound speed. Moreover, Geodesic Acoustic Modes (GAMs) [49] match the same dispersion relation, $f_{B A E}$, as we approach the plasma core where zonal flows and kinetic effects are dominant [50]. Taking into account background diamagnetic effects, a reasonable agreement of the $f_{\text {Sierpes }}$ with the core $f_{B A E / G A M}$ given by Zonca et al [51] has been found when the fast-ion pressure is not too large [52]. However when the fast-ion pressure achieves values comparable to the plasma pressure, a $f_{\text {Sierpes }}$ chirp of up to $\Delta f \approx 40 \mathrm{kHz}$ occurs, indicating an EPM character like in the Energetic Particle GAM (EGAM) case [53, 54], not explained by the common BAE/GAM dispersion relation. A detailed analysis of the Sierpes mode internal structure, polarization and nonlinear frequency behaviour is necessary to elucidate the nature of the Sierpes mode $[55,56]$.

To estimate the contribution of the individual TAEs and Sierpes mode to the FIL pattern, a time window has been selected in which they do not change strongly in frequency and no influence from other MHD activity is detected. Fig.9(a) and (b) show, respectively, the spectrograms of the magnetic fluctuation and of the FIL at high energies during that time window. We track the frequencies of the TAEs, $n=4$ and $n=5$, and of the Sierpes mode in the FIL spectrogram and extract their amplitudes, as shown in Fig.9(c). Although each MHD fluctuation is ejecting ions during the entire time window, it is obvious that the FIL at the Sierpes frequency are stronger than the TAEs induced FIL. The correlation in the occurrence of the spikes from $t=1.39 \mathrm{~s}$ on in Fig.9(c) gives further evidence for a coupling between either MHD fluctuation [57] or FIL mechanisms. However, the frequency behavior of the magnetic perturbation for both modes reveals no coupling between MHD fluctuations; as a consequence only the losses are connected. The more virulent effect of the Sierpes Mode on the fast-ion population might be explained on the basis of a broader loss condition. 
The TAE and Sierpes radial structure has been reconstructed by means of high time resolution SXR measurements, see Fig.10, to understand the combined effect of both MHD instabilities on the fast-ion transport [58]. The maximum TAE displacement ranges from 0.1 to $0.4 \mathrm{~mm}$ and the inferred core magnetic fluctuation amounts to $\delta b_{r} / B_{t}=0.2-5 \times 10^{-4}$. The Sierpes mode has a more core-localized eigenfunction (Fig.10), which is peaked around $\rho_{p o l} \approx 0.25$ and it extends up to $\rho_{\text {pol }} \approx 0.5$, leading to a maximum displacement of the order of $0.5 \mathrm{~mm}$ in the plasmas analyzed so far. It is interesting to note that there is a radial region, $\rho_{\text {overlap }} \in(0.2,0.5)$, where the $n=4 \mathrm{TAE}$ and Sierpes eigenfunctions overlap with non-zero values. The overlapping of radial eigenfunctions might be the reason for the drastic increase in the FIL signal when both modes are present simultaneously, by channeling the ions which fulfill the loss conditions from the plasma core all the way to the edge. This channeling process is illustrated in Fig.10 where the ICRH fast hydrogen ion pressure profile, calculated with the PION code [59], has been superimposed [60].

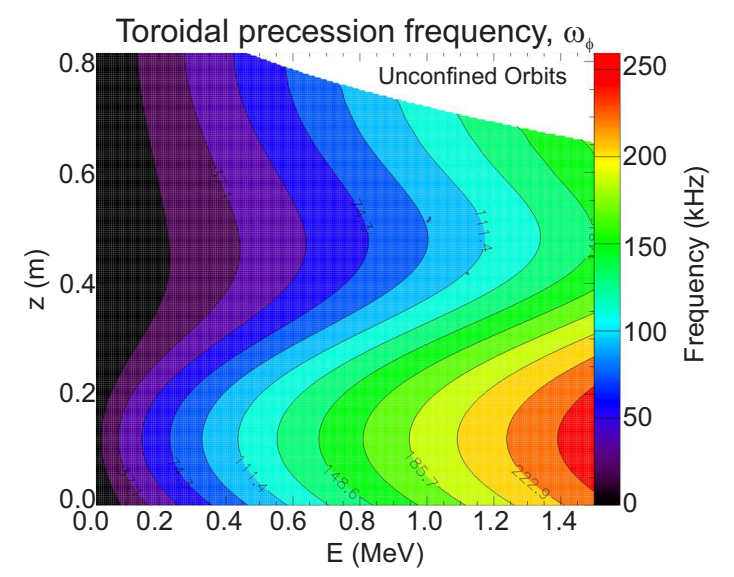

FIG. 11: AUG discharge \#21083: Toroidal precession frequencies of hydrogen ions with onaxis turning points described by $\Lambda=\mu B_{0} / E=1$. The vertical axis corresponds to the height of the turning point, $z\left(P_{\phi}\right)$, above the midplane.

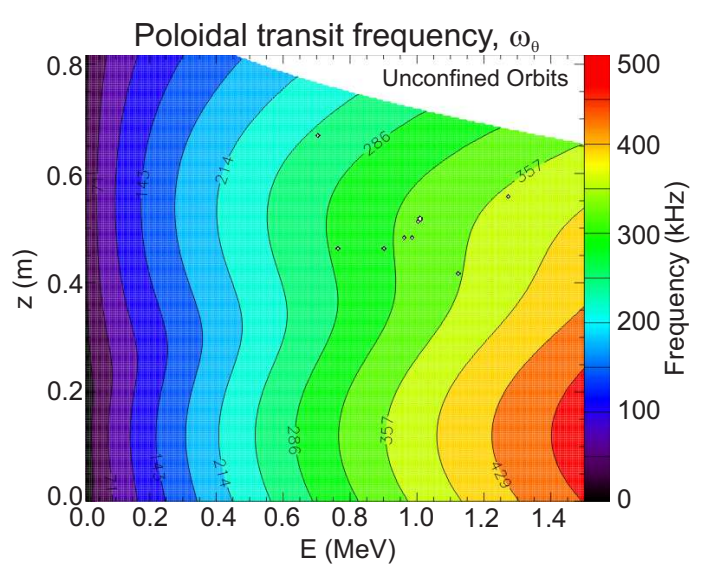

FIG. 12: AUG discharge \#21083: Poloidal transit frequencies of hydrogen ions with on-axis turning points described by $\Lambda=\mu B_{0} / E=1$. The vertical axis corresponds to the height of the turning point, $z\left(P_{\phi}\right)$, above the midplane.

The FIL mechanisms due to TAEs and Sierpes mode are investigated using the HAGIS code [24], v8.09, and will be the subject of a forthcoming detailed publication. Fast-ion orbits are 
usually described by their constant of motion; energy, pitch angle and toroidal canonical momentum, $\left(E, \Lambda, P_{\phi}\right)$. A simplified RF particle distribution function for on-axis ICRF minority heating has been simulated by taking pitch angle $\Lambda=\frac{\mu B_{0}}{E}=1$. This corresponds to trapped orbits with turning points along the ICRH resonance layer, a vertical line passing through the magnetic axis. The toroidal precession frequencies, $\omega_{\phi}$, and the poloidal transit frequencies, $\omega_{\theta}$, of a set of particles with pitch angle, $\Lambda=1$, and energies in the range measured by FILD, up to $1.5 \mathrm{MeV}$, are displayed in Fig.11 and Fig.12, respectively. In this special case, on-axis ICRF minority heating, the use of $z$ and $P_{\phi}$ as the phase-space variable is equivalent at the turning points $\left(v_{\|}=0\right)$, where $P_{\phi}=R m_{i} v_{\|}-Z e \psi_{p}(z)=-Z e \psi_{p}(z)$, with $R$, the magnetic axis radius, $m_{i}$, the fast-ion mass, $Z$, the atomic number, $e$, the electron charge and $\psi_{p}$, the poloidal flux. It is important to note that in AUG ICRF heated plasmas typical orbital frequencies of fast-ions from the tail of the ICRH distribution function, $\omega_{\phi}$ and $\omega_{\theta}$, are of the same order of typical Alfvenic frequencies, $f_{T A E s} \approx 200 \mathrm{kHz}$.
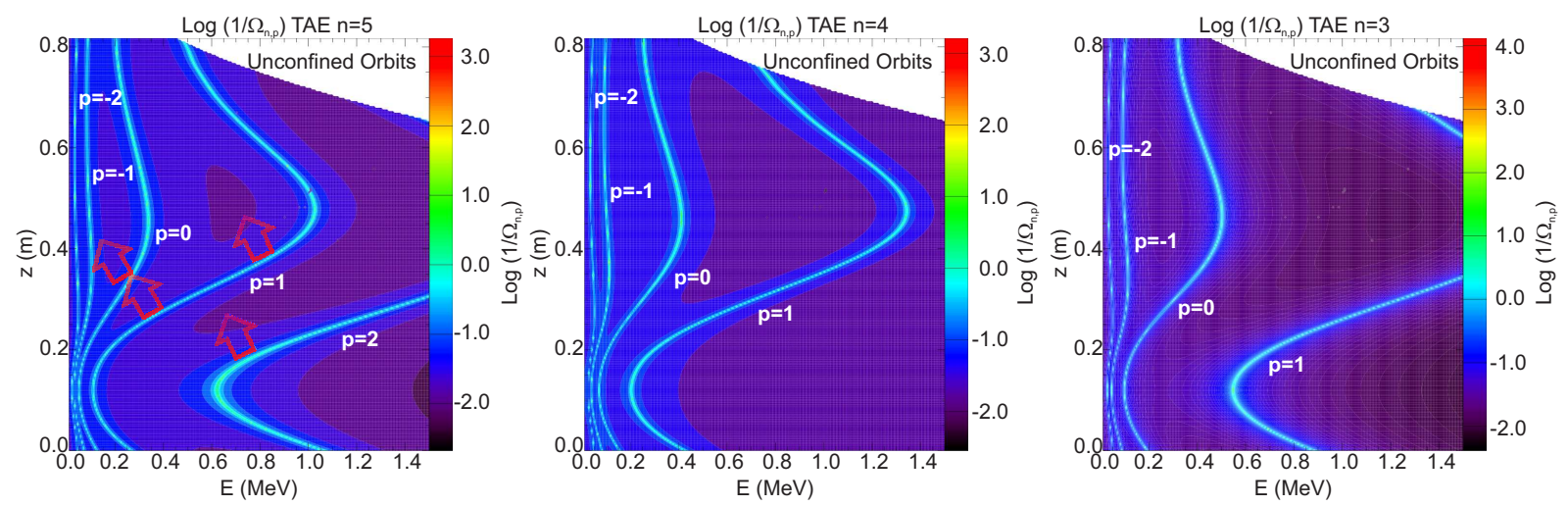

FIG. 13: AUG discharge \#21083: Phase-space resonance lines between on-axis ICRF heated hydrogen ions and the $n=5, n=4$ and $n=3$ TAEs $(a),(b)$ and $(c)$ respectively. Red arrows in the left panel illustrate the possible exchange of particles between adjacent resonances.

A resonant wave-particle interaction takes place if the resonance condition introduced in the previous section, $\Omega_{n, p}=n \omega_{\phi}-p \omega_{\theta}-\omega \approx 0$, is fulfilled. By plotting, $\log \left(1 / \Omega_{n, p}\right)$ in the energy range of the fast-ions measured by FILD, we can identify the regions of phase-space where a resonant interaction could occur [34]. The resonance condition fulfilled by the characteristic trapped fast-ion orbit frequencies and the mode rotation frequency leads to a phase matching between fast-ion orbit and TAE fluctuation. The banana tips of a resonant trapped fast-ion drift radially due to the $E \times B$-drift (with $E$ the poloidal electric field induced by the high-frequency MHD fluctuation) with a net inwards or outwards drift depending on the relative phase between MHD fluctuation and particle movement. Fig.13 contour plots show the on-axis ICRF heated hydrogen ions that are resonant with the $n=3, n=4$ and $n=5$ TAEs for the AUG plasma discharge \#21083. The wave-particle interaction results in an exchange of energy, $E$, and toroidal canonical momentum, $P_{\phi}$ which at the tips of the bananas translates into a radial drift of the particle. In Fig.13, this particle drift in phase-space is illustrated by red arrows on the left panel. Each resonance condition may then serve as particle seed for the adjacent one, leading to a fast-ion channeling which would be more effective in the case of fluctuations with multiple poloidal harmonics. By plotting all the possible resonance lines for the present TAEs, from $n=3$ up to $n=7$, with poloidal bounce harmonics, $p$, from $p=-2$ up to $p=3$, we observe that virtually all fast-ions with energies up to $1.5 \mathrm{MeV}$ may become resonant and interact with the TAEs. A non-linear simulation of the wave-particle interaction is necessary to identify the effective resonances. As Nazikian et al [61] reported recently from JET and DIII-D 
plasmas, we observe in Fig.13 and Fig.14(a) how sub-alfveninc fast-ions can also get trapped in the potential well of the wave field through resonance lines with negative poloidal bounce harmonics, $p$, driving the modes unstable. Fig.14(b) shows the resonance lines between the fast-ions and the Sierpes mode $n=4$ observed in plasma. The particle-Sierpes main resonance, $p=1$, appears at mid heights, covering the whole energy range up to $1 \mathrm{MeV}$, and so crossing most of the TAE resonance lines of Fig.14(a). A spatial overlapping of Sierpes and TAE eigenfunction enables the channeling of fast-ions in phase-space as well as the intersection of resonance lines and so a possible fast-ion avalanche in stochastic orbits. This fast-particle channeling in phase-space may be the responsible not only for the high FIL fluxes when both instabilities are present at the same time, but also for the driving of the instabilities due to the modification of fast-ion distribution function gradients. A quantitative analysis of the fast-ion channeling due to a radial chain of MHD fluctuations is under way to predict its impact in ITER.
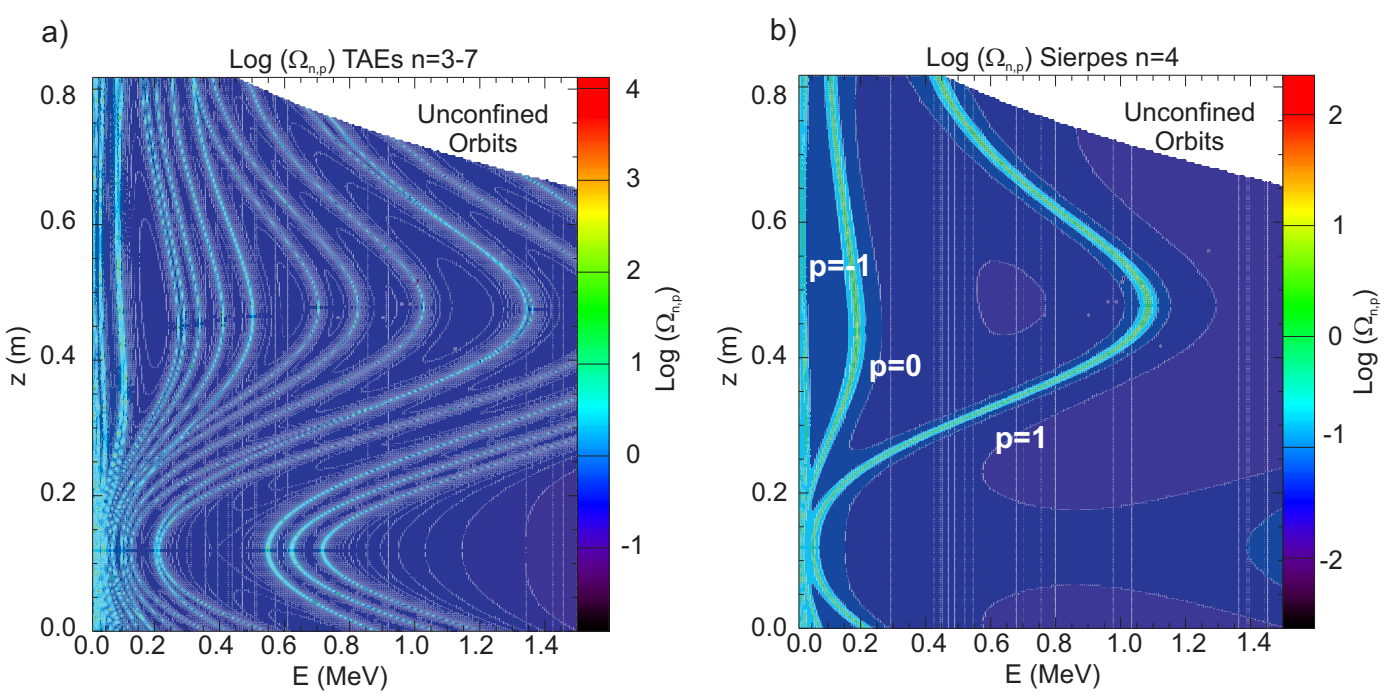

FIG. 14: AUG discharge \#21083: Phase-space resonance lines between on-axis ICRF heated hydrogen ions and; (a) TAEs with $n=3-7$ for the poloidal bounce harmonics from $p=-2$ up to $p=3$, and $(b)$ the Sierpes mode $n=4$ with poloidal bounce harmonics ranging from $p=-1$ up to $p=1$.

\section{Summary and conclusions}

In summary, we have develop a scintillator based detector for MHD induced fast-ion losses which allows to investigate the fast-ion losses through spectrograms. This new technique makes possible to evaluate the effect of each MHD fluctuation on the fast-ion loss (FIL) in terms of lost ion energy, pitch angle and flux. This information is crucial to understand the interplay between MHD fluctuations and fast-ions and their possible resonances. In the framework of these experiments, we have obtained, and studied in detail, time-resolved energy and pitch angle measurements of FIL caused by NTMs and TAEs. NTM magnetic islands induce FIL of both, NBI and ICRH origin. In the case of NBI fast-ions, their NTM induced losses are explained as a result of the drift islands formed by the fast-ions in particle phase-space. An eventual overlapping of these drift islands leads to an orbit stochasticity and therefore to an enhancement of the FIL. On the other hand, the loss of ICRH fast-ions is explained by a waveparticle resonant interaction through the wave and fast-ion orbital frequencies. The analysis of FIL due to TAEs has revealed the existence of a new core localized MHD fluctuation in 
AUG, the Sierpes mode. The Sierpes mode is a non-pure Alfvenic fluctuation which appears in the acoustic branch, dominating the transport of fast-ions in ICRF heated discharges. To understand the effect of both fluctuations, TAEs and Sierpes, on the fast-ion transport, their radial structures have been reconstructed by means of high time resolution SXR measurements. A spatial overlapping of their eigenfunctions leads to a fast-ion loss coupling, showing the strong influence that a core-localized MHD fluctuation may have on the TAE induced fast-ion transport. In general, a radial chain of MHD fluctuations with overlapping eigenfunctions may lead to a channeling of fast-ions in the particle phase-space causing severe consequences on the plasma facing components.

Further efforts are needed to evaluate the internal redistribution and possible loss of fast-ions due to individual and radial chains of MHD fluctuations. This redistribution may, in fact, affect considerably the fast-ion drive itself and modify the MHD activity. The theoretical explanation of this fast-ion channeling process is a challenge for the present codes and computing resources which implies the simultaneous treatment of different MHD fluctuations and fast-ion populations. The large fast-ion pressure expected in a burning plasma makes necessary this extra effort to extrapolate to ITER the results presented here, avoiding grave consequences.

\section{Acknowledgments}

The first author wishes to acknowledge the support of F. Ryter and the fruitful discussions with G. D. Conway and A. G. Elfimov (University of Sao Paulo, Brazil) on the properties of geodesic acoustic modes.

\section{References}

[1] SHIMADA, M. et al., Nucl. Fusion 47 (2007) S1.

[2] FASOLI, A. et al., Nucl. Fusion 47 (2007) S264.

[3] GARCIA-MUNOZ, M. et al., Nucl. Fusion 47 (2007) L10.

[4] ZWEBEN, S. J., Nucl. Fusion 29 (1989) 825.

[5] ISOBE, M., Rev. Sci. Instrum. 70 (1999) 827.

[6] WERNER, A. et al., Rev. Sci. Instrum. 72 (2001) 780.

[7] NISHIURA, M. et al., Rev. Sci. Instrum. 75 (2004) 3646.

[8] BAEUMEL, S. et al., Rev. Sci. Instrum. 75 (2004) 3563.

[9] DARROW, D. S., Rev. Sci. Instrum. 79 (2008) 023502.

[10] JIMENEZ-REY, D. et al., Rev. Sci. Instrum. 79 (2008) 093511.

[11] GUENTER, S. et al., Nucl. Fusion 47 (2007) 920.

[12] POLI, E. et al., Phys. Plasmas 15 (2008) 032501.

[13] GARCIA-MUNOZ, M. et al., Phys. Rev. Lett. 100 (2008) 055005.

[14] HEIDBRINK, W. W. et al., Phys. Rev. Lett. 99 (2007) 245002.

[15] PANKIN, A. et al., Comp. Phys. Comm. 159 (2004) 157.

[16] DARROW, D. S. et al., Nucl. Fusion 37 (1997) 939. 
[17] ZWEBEN, S. J. et al., Nucl. Fusion 40 (2000) 91.

[18] CAROLIPIO, E. M. et al., Nucl. Fusion 42 (2002) 853.

[19] FOREST, C. B. et al., Phys. Rev. Lett. 79 (1997) 427.

[20] WHITE, R. B., Phys. Fluids 27 (1984) 2455.

[21] GOURDON, C., Programme optimise de calculs numeriques dans les configurations magnetiques, Centre d'etudes nucleaires de Fontenay aux Roses, 1970.

[22] STRUMBERGER, E. et al., New J. Phys. 10 (2008) 023017.

[23] MYNICK, H. E., Phys. Fluids B 5 (1993) 1471.

[24] PINCHES, S. D., Comput. Phys. Commun. 111 (1998) 131.

[25] WHITE, R. B. et al., Phys. Fluids 26 (1983) 2958.

[26] HEIDBRINK, W. W., Phys. Plasmas 15 (2008) 055501.

[27] FU, G. Y. and VAN-DAM, J. W., Phys. Fluids B 1 (1989) 1949.

[28] DiPPOlito, A. and GOEDBLOED, J. P., Plasma Phys. 22 (1980) 1091.

[29] WONG, K. L. et al., Phys. Rev. Lett. 66 (1991) 1874.

[30] CHEN, L., Phys. Plasmas 1 (1994) 1519.

[31] SNIPES, J. et al., Nucl. Fusion 46 (2006) 1036.

[32] LAUBER, P. and GUENTER, S., Nucl. Fusion 48 (2008) 084002 (9pp).

[33] VLAD, G. et al., Nucl. Fusion 46 (2006) 1.

[34] PINCHES, S. D. et al., Nucl. Fusion 46 (2006) S904.

[35] FREDRICKSON, E. et al., Nucl. Fusion 46 (2006) S926.

[36] ISHIKAWA, M. et al., Nucl. Fusion 46 (2006) S898.

[37] SHINOHARA, K. et al., Nucl. Fusion 41 (2001) 603.

[38] BERNABEI, S. et al., Phys. Rev. Lett. 84 (2000) 1212.

[39] ISOBE, M. et al., Nucl. Fusion 46 (2006) S918.

[40] DUONG, H. et al., Nucl. Fusion 33 (1993) 749.

[41] VAN-ZEELAND, M. A. et al., Phys. Rev. Lett. 97 (2006) 135001.

[42] DARROW, D. et al., Nucl. Fusion 48 (2008) 084004 (6pp).

[43] BORBA, D. et al., Plasma Phys. Control. Fusion 46 (2004) 809.

[44] DA GRACA, S. et al., Plasma Phys. Control. Fusion 49 (2007) 1849.

[45] MCGUIRE, K. et al., Phys. Rev. Lett. 50 (1983) 891.

[46] TURNBULL, A. D. et al., Phys. Fluids B: Plasma Phys. 5 (1993) 2546. 
[47] HEIDBRINK, W. W. et al., Phys. Rev. Lett. 71 (1993) 855.

[48] GORELENKOV, N. et al., Phys. Lett. A 370 (2007) 70.

[49] CONWAY, G. D. et al., Plasma Phys. Control. Fusion 47 (2005) 1165.

[50] ZONCA, F. and CHEN, L., EPL (Europhysics Letters) 83 (2008) 35001 (6pp).

[51] ZONCA, F. et al., Plasma Phys. Control. Fusion 38 (1996) 2011.

[52] LAUBER, P. et al., 35 $5^{\text {th }}$ EPS Conference on Plasma Physics, Hersonissos, Crete, 2008 .

[53] NAZIKIAN, R. et al., Phys. Rev. Lett. 101 (2008) 185001.

[54] FU, G. Y., Phys. Rev. Lett. 101 (2008) 185002.

[55] SMOLYAKOV, A. I. et al., Plasma Phys. Control. Fusion 50 (2008) 115008 (15pp).

[56] ELFIMOV, A. G., Phys. Plasmas 15 (2008) 074503.

[57] CROCKER, N. A. et al., Phys. Rev. Lett. 97 (2006) 045002.

[58] PIOVESAN, P. et al., Nucl Fusion 48 (2008) 065001.

[59] ERIKSSON, L.-G. et al., Nucl. Fusion 33 (1993) 1037.

[60] MANTSINEN, M. J. et al., Analysis of icrf-accelerated ions in asdex upgrade, volume 933, pages 99-102, AIP, 2007.

[61] NAZIKIAN, R. et al., Phys. Plasmas 15 (2008) 056107. 